\title{
On electron acceleration at CIR related shock waves
}

\author{
G. Mann ${ }^{1}$, H. T. Classen ${ }^{1}$, E. Keppler ${ }^{2}$, and E. C. Roelof ${ }^{3}$ \\ 1 Astrophysikalisches Institut Potsdam, An der Sternwarte 16, 14482 Potsdam, Germany \\ 2 Max-Planck-Institut für Aeronomie, Max-Planck-Str. 2, 37191 Katlenburg-Lindau, Germany \\ 3 Applied Physics Laboratory, John Hopkins University, Laurel, MD 20723, USA
}

Received 15 March 2002 / Accepted 15 May 2002

\begin{abstract}
The interaction of fast and slow speed solar wind streams leads to the formation of so-called corotating interaction regions (CIRs) in the heliosphere. These CIRs are often associated with shock waves, at which electrons are accelerated as observed by the Ulysses spacecraft. A correlation between the ratio of energetic electron fluxes at the crossing of CIR related shocks to those in the far upstream region of these shocks and the magnetic field compression of the associated shocks has been revealed by analysing the data of the HISCALE instrument aboard Ulysses. This result can be explained by a model of electron acceleration at shock waves, where the electrons gain energy due to multiple reflections at large amplitude magnetic field fluctuations occurring in the vicinity of the shock transition.
\end{abstract}

Key words. acceleration of particles - sun: solar wind - shock waves

\section{Introduction}

The Sun is a source of a permanent stream of charged particles (e.g. electrons, protons and heavy ions) penetrating into the interplanetary space. It is the so-called solar wind forming the heliosphere due to its interaction with the interstellar wind (Parker 1958). The solar wind was originally discovered by in-situ measurements of the Mariner 2 spacecraft in 1962 (Neugebauer 1966). It is temporally and spatially structured (see Schwenn 1990 as a review). There are high and slow speed solar wind streams. The coronal holes with open magnetic field structures are the sources of the high speed streams, whereas the slow solar wind is coming from regions with closed magnetic field structures located around the equatorial plane (Schwenn 1990). Due to the rotation of the Sun the fast and slow solar wind streams interact with each other, leading to the formation of a so-called corotating interaction region (CIR) (Pizzo 1978). An interface is located within the CIR. It is a contact discontinuity dividing the fast solar wind plasma from that of the slow solar wind stream. In many cases a pair of forward and reverse shocks forms the boundaries of CIRs. The forward shocks are propagating into the slow solar wind towards the equatorial plane whereas the reverse shocks are travelling pole-ward into the fast solar wind stream (Gosling \& Pizzo 1999). The formation of CIRs mainly takes place at distances beyond $1 \mathrm{AU}$. Since the Ulysses spacecraft was exploring the three-dimensional heliosphere between 1 and $5 \mathrm{AU}$ during the declining period of solar activity (Marsden et al. 1996), the Ulysses mission is highly appropriate to study all phenomena related to CIRs.

Send offprint requests to: G. Mann, e-mail: GMann@aip.de
The shock waves associated with CIRs are able to generate energetic electrons, protons and heavy ions, as well-known from the observations of the Pioneer and Voyager spacecraft (McDonald et al. 1976; Barnes \& Simpson 1976). The basic features of energetic particles associated with CIRs have been summarized by Mason \& Sanderson (1999). As an example, Fig. 1 offers the energetic particle and plasma data recorded by the Ulysses spacecraft during its passage through the CIR No. 5 occurring during the days 282-286 in 1992. (The numbering of CIRs observed by Ulysses was originally introduced by Bame et al. 1993.) The plasma and magnetic field data were provided by the solar wind plasma instrument (SWOOPS) (Bame et al. 1992) and the VHM/FGM magnetometer (Balogh et al. 1992) aboard Ulysses, respectively. The location of CIR is evidently identified in the data of the solar wind speed and magnitude of the magnetic field as indicated by the dashed vertical lines in Fig. 1. The forward and reverse shocks have Alfvén-Mach numbers $M_{\mathrm{A}}=1.42$ and $M_{\mathrm{A}}=3.0$ as well as jumps of the magnetic field $B_{2} / B_{1}=1.40$ and $B_{2} / B_{1}=2.30$ (Balogh et al. 1995; Classen et al. 1998), respectively. Here, $B_{1}$ and $B_{2}$ denote the up- and downstream magnetic field of the shock, respectively. The two panels at the top of Fig. 1 present the proton and electron fluxes in different channels covering the range $0.61-19 \mathrm{MeV}$ and $38-315 \mathrm{keV}$, respectively, as measured by the HISCALE (Lanzerotti et al. 1992) and the COSPIN instrument (Simpson et al. 1992) aboard Ulysses. Whereas the flux of energetic protons increases at few orders of magnitudes at both the forward and reverse shock, the flux of energetic electrons is essentially more enhanced at the reverse shock (see Fig. 1). The evolution of energetic electrons and ions has been discussed by Simnett \& Roelof (1995) 

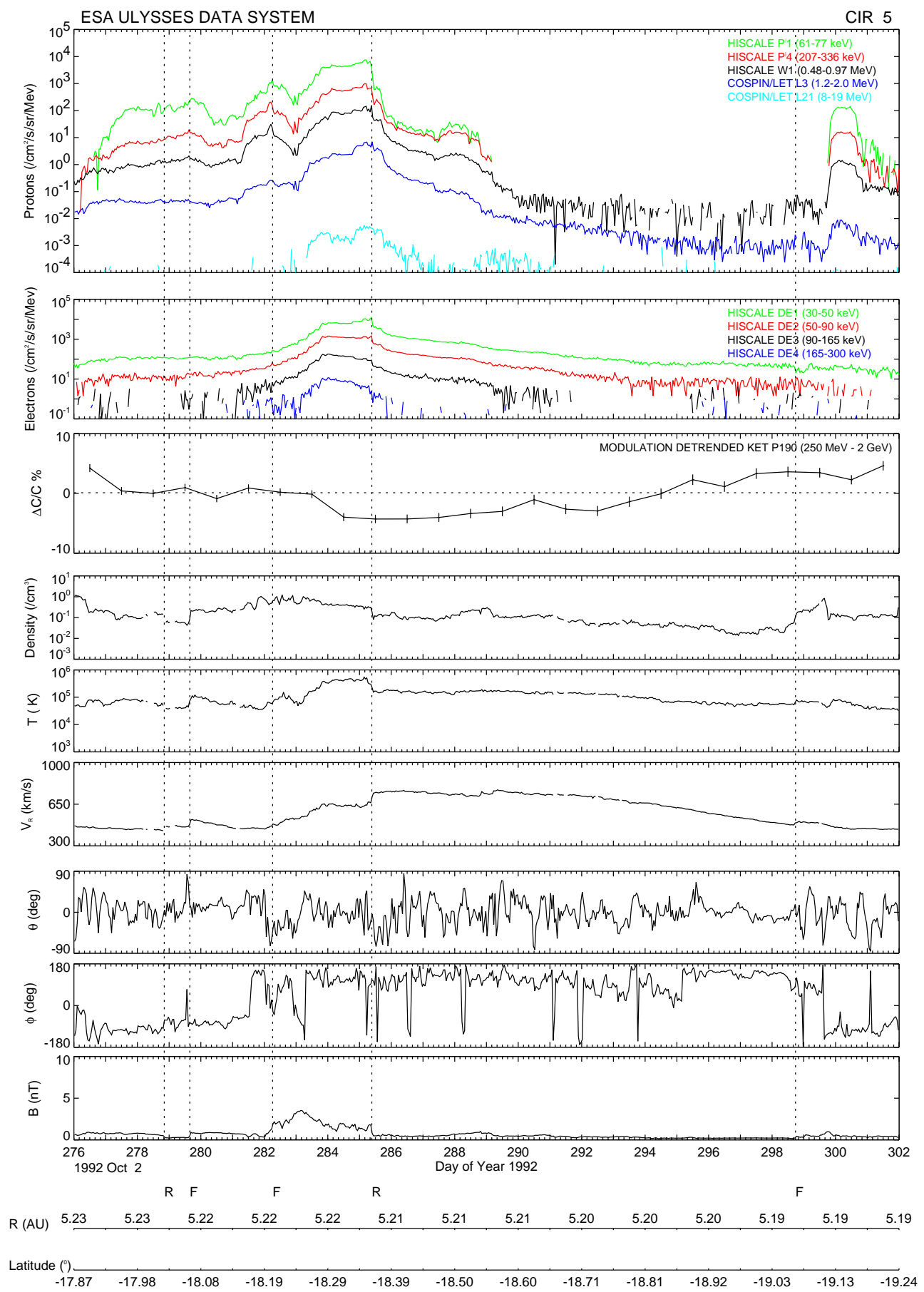

Fig. 1. HISCALE data of energetic protons (top panel) and electrons (2nd panel from top) as well as the behaviour of the proton number density $N$ (4th panel), the proton temperature $T$ (5th panel), the solar wind velocity $v_{\mathrm{sw}}$ (6th panel) and the magnetic field in polar coordinates (three panels at the bottom) during the passage of the CIR No. 5 by the ULYSSES spacecraft.

and Roelof et al. (1996) for the period of the first southbound journey of the Ulysses spacecraft. At low heliospheric latitudes both energetic electrons and ions were simultaneously seen with the occurrence of the forward (FS) and reverse (RS) shocks (Keppler et al. 1996). The last shock pair (CIR No. 15) was observed at a distance of $4.58 \mathrm{AU}$ and a latitude of $-33.7^{\circ}$, while the reverse shocks were directly seen by Ulysses for several more solar rotations (Gosling et al. 1993). The relationship between particle acceleration at CIR-related shocks and their magnetohydrodynamic (MHD) parameters was studied by Desai et al. (1998) and Classen et al. (1998). Classen et al. (1998) analysed a sample of 18 CIRs associated with 33 shocks. They found that shocks accelerate predominantly electrons and protons if the Alfvén-Mach number $M_{\mathrm{A}}$ and the angle $\theta_{B, n}$ between the upstream magnetic field and the shock normal simultaneously fulfilled the conditions $M_{\mathrm{A}}>2.5$ and $50^{\circ} \leq \theta_{B, n} \leq 75^{\circ}$. 
The aim of the present paper is to investigate the efficiency of electron acceleration at CIR related shocks (see Mann 1999 for a preliminary study). Note that the generation of energetic electrons has also been observed at shocks in the solar corona (Wild \& McCready 1950; Cane et al. 1981; Cairns \& Robinson 1987), at travelling interplanetary shocks (Tsurutani \& Lin 1985; Lopate 1989), and at the Earth's bow shock (Anderson et al. 1969; Scarf et al. 1971). The electron fluxes used in the present paper has been provided by the HISCALE instrument (Lanzerotti et al. 1992) in the range $30-50 \mathrm{keV}$ aboard the Ulysses spacecraft. The electron fluxes $j_{\text {shock }}$ at the shock crossing are compared with those $j_{0}$ measured during quiet solar wind periods before and after the CIR (see Sect. 2). Furthermore, the ratios $j_{\text {shock }} / j_{0}$ are related to the magnetic field compression $B_{2} / B_{1}$ of the associated shock. Mann \& Classen (1995) proposed a mechanism of generation of energetic electrons at collisionless shocks. It is well-known that super-critical shocks are accompanied with large amplitude magnetic field fluctuations in the vicinity of the shock transition (Kennel et al. 1985). Electrons can be reflected and subsequently accelerated at these fluctuations. Due to multiple encounters of electrons with these fluctuations they receive a considerable acceleration (Mann \& Classen 1995). This special acceleration mechanism will be introduced in a quantitative manner in Sect. 3, and subsequently adopted to explain the relations between the flux ratios $j_{\text {shock }} / j_{0}$ and jumps of the magnetic field $B_{2} / B_{1}$ of the associated shocks in Sect. 4.

\section{Observations}

The HISCALE instrument (Lanzerotti et al. 1992) aboard Ulysses is able to measure the fluxes of energetic electrons in four different channels, i.e. DE1: $30-50 \mathrm{keV}$, DE2: 50-90 keV, DE3: $90-165 \mathrm{keV}$, and DE4: $165-300 \mathrm{keV}$. The data recorded in the channel DE1 are employed to compare the electron fluxes $j_{\text {shock }}$ at the shock crossing with those $j_{0}$, which are determined during quiet conditions of the solar wind stream related with the corresponding shock. It should be recalled, that the forward and reverse shocks are travelling into the slow and fast speed solar wind (Pizzo 1978), respectively.

The data analysis can be demonstrated for example in Fig. 1. The energetic electron fluxes $j_{0}$ of quiet solar wind conditions are chosen to be at day 281 and 293 for the forward and reverse shock in this particular case, respectively. These days have been chosen because the particle, plasma and magnetic field data show no strong and rapid changes, i.e. there were really quiet solar wind conditions.

The results of the whole data analysis are summarized in Table 1. CIRs Nos. 1-18 (Bame et al. 1993) have been employed for this study. But only a sub-sample of 15 CIR related shocks could be used for investigating the efficiency of electron acceleration, since the energetic electron fluxes $j_{\text {shock }}$ and $j_{0}$ can reliably determined only at these shocks presented in Table 1. The parameters of the associated shocks were given by Balogh et al. (1995) and Classen et al. (1998). $N_{1}, T_{1}, N_{2}$, and $T_{2}$ are the particle number densities and temperatures in the up- and downstream region, respectively. The upstream

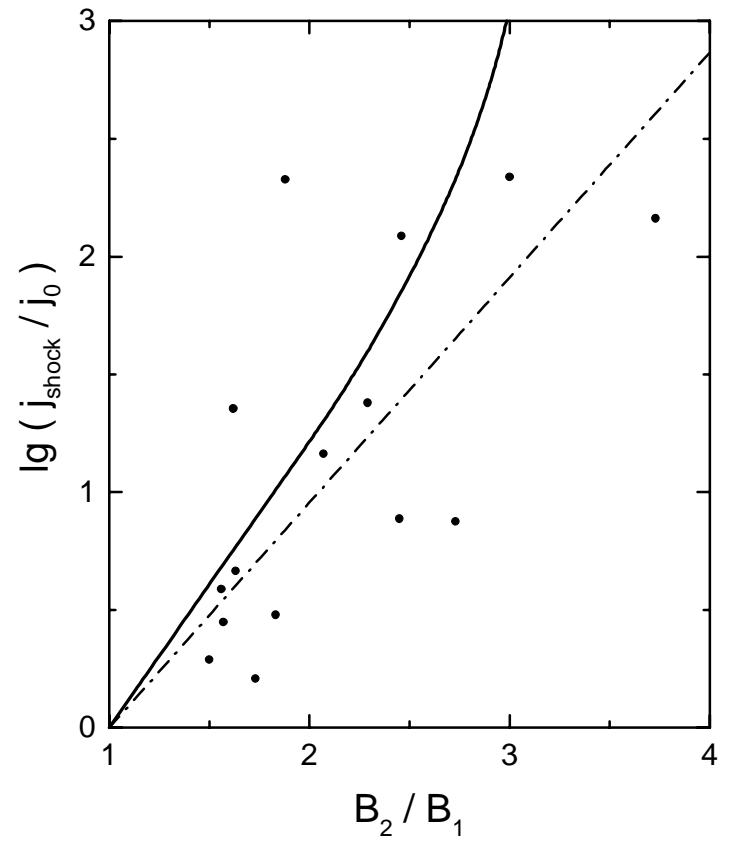

Fig. 2. Correlation between the ratios $\lg \left(j_{\text {shock }} / j_{0}\right)$ and the jump of the magnetic field $B_{2} / B_{1}$ for $15 \mathrm{CIR}$ related shocks (Table 1). The full line represents the theoretical result obtained by means of Eq. (22). The dashed-dotted line shows the line of linear regression.

regions of the CIR related shocks (Classen et al. 1998) have the mean values of the following plasma parameters:

$$
\begin{array}{ll}
\text { upstream magnetic field } & B_{1}=0.9 n T \\
\text { upstream density } & N_{1}=0.25 \mathrm{~cm}^{-3} \\
\text { upstream temperature } & T_{1}=0.9 \times 10^{5} \mathrm{~K} \\
\text { upstream plasma beta } & \beta_{1}=0.8 .
\end{array}
$$

These values will be used as typical upstream parameters of CIR related shocks in Sect. 4. The angle $\theta_{B, n}$ between the upstream magnetic field and the shock normal varies between $19^{\circ}-82^{\circ}$ with a mean value of $54^{\circ}$ for these shocks (Classen et al. 1998), i.e. CIR related shocks are mainly quasiperpendicular, $\left(\theta_{B, n}>45^{\circ}\right)$. The correlation between the ratios $\lg \left(j_{\text {shock }} / j_{0}\right)$ and the jump of the magnetic field $B_{2} / B_{1}$ is depicted for all 15 CIR related shocks (Table 1) in Fig. 2. The dashed dotted line represents the linear regression with a correlation coefficient of 0.649 and a standard deviation of 0.594 . Therefore, the efficiency of electron acceleration expressed by the ratios $\lg \left(j_{\text {shock }} / j_{0}\right)$ is increasing for shocks with a stronger jump of the magnetic field $B_{2} / B_{1}$ (Fig. 2). This result can be explained by a model of electron acceleration at collisionless shocks as described in Sect. 3.

\section{Mirror acceleration}

As already mentioned the CIR related shocks are usually quasiperpendicular, i.e. $\theta_{B, n}>45^{\circ}$. For a plasma-beta $\beta \approx 0.8$ such shocks are super-critical, if their Alfvén-Mach number is greater than 2.0 (Kennel et al. 1985). That is mostly the case of the shocks considered in this paper (see Table 1). Figure 3 shows the behaviour of the magnitude of the magnetic field during the crossing of the CIR related shock No. 7F as measured 
Table 1. Energetic electron fluxes $(30-50 \mathrm{keV})$ at the shock crossing and at quiet solar wind conditions as well as the corresponding parameters of the CIR related shocks.

\begin{tabular}{ccccccc}
\hline \hline$N o$. & $j_{\text {shock}}\left[1 / \mathrm{cm}^{2} \cdot \mathrm{s} \cdot \mathrm{sr} \cdot \mathrm{MeV}\right]$ & $j_{0}\left[1 / \mathrm{cm}^{2} \cdot \mathrm{s} \cdot \mathrm{sr} \cdot \mathrm{MeV}\right]$ & $\log \left(j_{\text {shock }} / j_{0}\right)$ & $B_{2} / B_{1}$ & $M_{\mathrm{A}}$ & $T_{2} / T_{1}$ \\
\hline $1 R$ & $5.06 \times 10^{2}$ & $3.13 \times 10^{2}$ & 0.208 & 1.73 & 1.9 & 1.4 \\
$3 R_{1}$ & $2.26 \times 10^{3}$ & $1.00 \times 10^{2}$ & 1.354 & 1.62 & 1.8 & 1.7 \\
$4 R$ & $8.30 \times 10^{3}$ & $5.71 \times 10^{1}$ & 2.162 & 3.73 & 5.3 & 3.8 \\
$5 F$ & $2.41 \times 10^{2}$ & $1.24 \times 10^{2}$ & 0.289 & 1.50 & 1.4 & 1.7 \\
$5 R$ & $9.90 \times 10^{3}$ & $8.08 \times 10^{1}$ & 2.088 & 2.46 & 3.0 & 3.0 \\
$6 F$ & $6.47 \times 10^{3}$ & $3.05 \times 10^{1}$ & 2.327 & 1.88 & 2.4 & 4.0 \\
$7 F$ & $4.61 \times 10^{2}$ & $1.53 \times 10^{2}$ & 0.479 & 1.83 & 3.0 & 5.0 \\
$7 R$ & $2.31 \times 10^{4}$ & $1.06 \times 10^{2}$ & 2.338 & 3.00 & - & 3.3 \\
$8 F$ & $4.39 \times 10^{2}$ & $1.13 \times 10^{2}$ & 0.589 & 1.56 & 1.7 & 2.5 \\
$8 R$ & $1.23 \times 10^{4}$ & $1.60 \times 10^{3}$ & 0.886 & 2.45 & 2.7 & 4.4 \\
$9 F$ & $4.95 \times 10^{2}$ & $1.77 \times 10^{2}$ & 0.447 & 1.57 & 2.5 & 2.0 \\
$10 R$ & $1.15 \times 10^{3}$ & $1.53 \times 10^{2}$ & 0.876 & 2.73 & 3.0 & 4.4 \\
$11 R$ & $8.84 \times 10^{2}$ & $1.91 \times 10^{2}$ & 0.665 & 1.63 & 2.4 & 1.2 \\
$13 R$ & $8.16 \times 10^{2}$ & $5.61 \times 10^{1}$ & 1.163 & 2.07 & 3.1 & 1.5 \\
$15 R$ & $1.15 \times 10^{3}$ & $4.80 \times 10^{1}$ & 1.379 & 2.29 & 2.8 & 1.7 \\
\hline
\end{tabular}

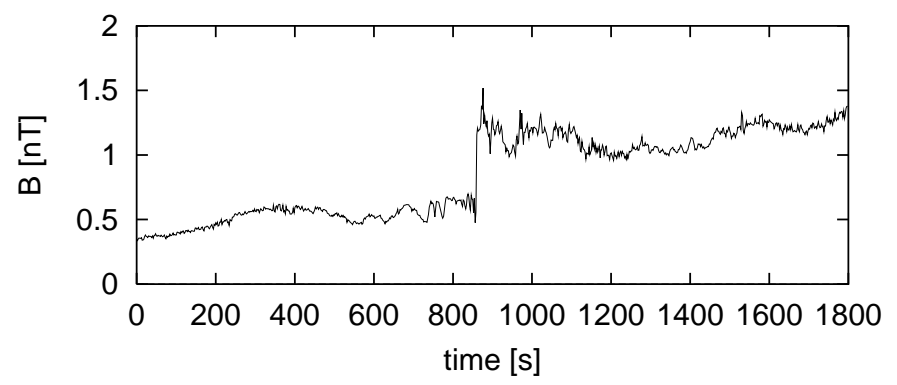

Fig. 3. Behaviour of the magnitude of the magnetic field during the crossing of the CIR related shock No. 7F as measured by the magnetometer aboard the ULYSSES spacecraft.

by the magnetometer (Balogh et al. 1992) aboard the Ulysses spacecraft. As seen in Fig. 3 quasi-perpendicular, super-critical shocks are accompanied with strong magnetic field fluctuations, especially in the downstream region. Furthermore, they show a so-called overshoot at the shock transition, i.e., the magnetic field compression $B_{\max } / B_{1}$ is locally stronger than the jump $B_{2} / B_{1}$ of the magnetic field according to the RankineHugoniot relations (Kennel et al. 1985), i.e., $B_{\max } / B_{1}>$ $\left(B_{2} / B_{1}\right)_{\mathrm{RH}}$, where $B_{\max }$ denotes the maximum magnitude of the magnetic field. These large amplitude magnetic field fluctuations can be considered as nonlinear magnetohydrodynamic waves. Such waves have the property that their velocity $V_{\text {wave }}$ is a monotonically increasing function of the magnetic field compression $B_{\max } / B_{0}$ (Mann 1995) where $B_{0}$ denotes the magnitude of the undisturbed ambient magnetic field. Thus, two neighbouring magnetic field fluctuations with different magnetic field compressions have consequently a non-vanishing relative velocity. They are establishing a system of converging magnetic mirrors, which are able to accelerate particles, as originally proposed by Fermi (1949).

Now, the movement of an electron between two neighbouring mirrors is considered in detail, following the way described by Chen (1984) and Mann \& Classen (1995). The computations are lengthy, but straightforward. Thus, only the main



Fig. 4. Scheme of converging magnetic mirrors $M 1$ and $M 2$.

line of thought will be presented. The mirrors $M 1$ and $M 2$ (see Fig. 4) are accompanied with magnetic field compressions $B_{M 1}$ and $B_{M 2}$ with $B_{M 1}>B_{M 2}$ and have the velocities $V_{1}$ and $V_{2}$ with $V_{1}>V_{2}$, respectively. Since energetic electrons with an energy of about $40 \mathrm{keV}$ are considered, this treatment can be done in a nonrelativistic manner, i.e. the required relation $\epsilon=E_{\text {kin }} / m_{\mathrm{e}} c^{2}=0.08 \ll 1\left(E_{\mathrm{kin}}\right.$, kinetic energy; $m_{\mathrm{e}}$, electron mass; $c$, velocity of light) is well fulfilled. The two mirrors are initially separated by a distance $L_{n}$ (see Fig. 4). The electron with an initial velocity $V_{n}$ parallel to the ambient magnetic field and a pitch angle $\alpha_{n}$ starts at the mirror $M 1$ and moves towards the mirror $M 2$, which is reached after a distance $L_{n}^{*}=L_{n}-\left(V_{1}-V_{2}\right) \Delta t_{n}^{*}$ and a time $\Delta t_{n}^{*}=L_{n} /\left(V_{n}-V_{2}\right)$ (see Fig. 4). At the mirror $M 2$ it is reflected and, subsequently, it returns towards the mirror $M 1$ with the velocity

$V_{n}^{*}=V_{n}-\Delta V_{2}$

parallel to the magnetic field. The velocity gain $\Delta V_{2}=2 V_{2}$. $\sec \theta_{2}$ results from the shock drift acceleration at the mirror $M 2$ (Toptygin 1980; Holman \& Pesses 1983; Decker 1988). Here, $\theta_{2}$ denotes the angle between the undisturbed ambient magnetic field $\boldsymbol{B}_{0}$ and the propagation direction of the mirror $M 2$. 
During the reflection process at the mirror $M 2$ the pitch angle is changed according to

$\alpha_{n}^{*}=\arctan \left[\frac{V_{n} \cdot \tan \alpha_{n}}{V_{n}-\Delta V_{2}}\right]$.

After this reflection at the mirror $M 2$ the electron moves back towards the mirror $M 2$, which is reached after a time period of $\Delta t_{n}^{* *}=L_{n}^{*} /\left(V_{n}^{*}+V_{1}\right)$. There, the electron is reflected again and gets a final velocity

$V_{n+1}=V_{n}^{*}+\Delta V_{1}$

parallel to the magnetic field and a pitch angle

$\alpha_{n+1}=\arctan \left[\frac{V_{n}^{*} \cdot \tan \alpha_{n}^{*}}{V_{n}^{*}+\Delta V_{1}}\right]$.

Here, $\Delta V_{1}$ is given by $\Delta V_{1}=2 V_{1} \cdot \sec \theta_{1}$, where $\theta_{1}$ denotes the angle between the undisturbed ambient field and the propagation direction of the mirror $M 1$. Thus, the $n$th revolution lasts $\Delta t_{n}=\Delta t_{n}^{*}+\Delta t_{n}^{* *}$ whereas the distance between the two mirrors is diminished to $L_{n+1}=L_{n}-\left(V_{1}-V_{2}\right) \cdot \Delta t_{n}$ (see Fig. 4).

Concerning this process it is generally assumed that the electrons are adiabatically reflected at the magnetic field compressions acting as magnetic mirrors. This assumption is justified if the gyroradius $r_{\mathrm{L}}$ of the electron is essentially smaller than the characteristic length scale of the magnetic field compressions. This length scale is typically 10 ion inertial lengths (Mann et al. 1994). Since electrons in the energy range $30-50 \mathrm{keV}$ have a typical gyroradius of 1.3 ion inertial lengths under plasma conditions near CIRs (see Sect. 2), the required condition is well fulfilled in the case considered here.

Since the mirrors in terms of two neighbouring large amplitude magnetic field compressions cannot penetrate each other, the distance between them can be reduced only up to a minimum one $L_{\mathrm{f}}$. Consequently, the acceleration process is finished either if $L_{n+1}<L_{\mathrm{f}}$ or if the electron leaves the region between the mirrors, since the pitch angle $\alpha_{n+1}$ becomes $\alpha_{n+1}<\alpha_{\mathrm{f}}=\arctan \left[\left(B_{0} / B_{M 2}\right)^{1 / 2}\right]$. The movement of an electron between these two mirrors leads finally to a nonuniform acceleration as illustrated in Fig. 5. In this special example the particle has an initial velocity $V_{0}=114 v_{\mathrm{A}}=5 v_{\text {th,e }}\left(v_{\mathrm{A}}\right.$, Alfvén speed; $v_{\text {th,e }}$, thermal electron velocity) parallel to the ambient magnetic field and an initial pitch angle $\alpha_{0}=85^{\circ}$. The distance between the two mirrors is diminished from $L_{0}=50 d_{i}$ up to $L_{\mathrm{f}}=10 d_{\mathrm{i}}$. The mirrors $M 1$ and $M 2$ are moving with the velocities $V_{1}=2.4 v_{\mathrm{A}}$ and $V_{2}=1.7 v_{\mathrm{A}}$, respectively. The magnetic field compression at the mirror $M 2$ is assumed to be $B_{M 2} / B_{0}=2.8$ resulting in a final pitch angle $\alpha_{\mathrm{f}}=36.7^{\circ}$. Adopting $\theta_{1}=\theta_{2}=54^{\circ}$ as typical values for CIR related shocks, $\Delta V_{1}=8.17 v_{\mathrm{A}}$ and $\Delta V_{2}=5.78 v_{\mathrm{A}}$ is found. The result of the numerically evaluated Eqs. (1)-(4) is presented in Fig. 5. Here, the temporal coordinate is normalized to the inverse of the proton cyclotron frequency $\omega_{\mathrm{ci}}^{-1}$ leading to a normalization of the velocities with respect to the Alfvén speed $v_{\mathrm{A}}$. The particle is nonuniformly accelerated in a discrete manner up to a final velocity $V_{\mathrm{f}}=1650 v_{\mathrm{A}}$ during a time of $57 \omega_{\mathrm{ci}}$ corresponding to a final energy of $33 \mathrm{keV}$, where $v_{\mathrm{A}}=50 \mathrm{~km} \mathrm{~s}^{-1}$ and $v_{\text {th,e }}=1150 \mathrm{~km} \mathrm{~s}^{-1}$ have been used as typical values of

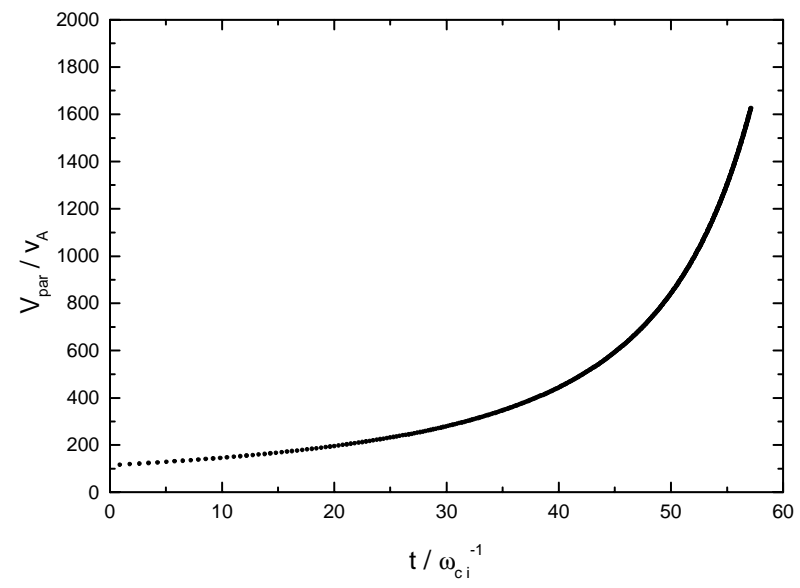

Fig. 5. Velocity-time diagram of a special example of the mirror acceleration. The chosen parameters are introduced in Sect. 3 . The velocity and the time are normalized to the Alfvén speed $v_{\mathrm{A}}$ and the inverse proton cyclotron frequency $\omega_{\mathrm{ci}}^{-1}$.

the Alfvén speed and thermal electron velocity in the quiet solar wind, respectively. These values correspond to the mean plasma parameters found in the upstream region of CIR related shocks (see Sect. 2).

Since the electrons move much faster than the mirrors, the acceleration process might be considered as a continuous one (see Fig. 5), i.e. the relationship

$V_{1}, V_{2}, \Delta V_{1}, \Delta V_{2} \ll V_{n}<c$

is well fulfilled for the cases under consideration. Then, the acceleration defined by $\Delta V_{n} / \Delta t_{n}$ with $\Delta V_{n}=V_{n+1}-V_{n}=\Delta V_{1}-$ $\Delta V_{2}=\Delta V_{\mathrm{G}}$ and $\Delta t_{n}=\Delta t_{n}^{*}+\Delta t_{n}^{* *}=2 L_{n} / V_{n}$ may be taken as a differential equation $\Delta V_{n} / \Delta t_{n} \Rightarrow \mathrm{d} V_{\|} / \mathrm{d} t$

$\frac{\mathrm{d} V_{\|}}{\mathrm{d} t}=\frac{\Delta V_{\mathrm{G}}}{2\left(L_{0}-\Delta V_{\mathrm{S}} t\right)} \cdot V_{\|}$

with the initial condition $V_{\|}(t=0)=V_{0}$. Here, $L_{n}=L_{0}-\Delta V_{\mathrm{S}} t$ with $\Delta V_{\mathrm{S}}=V_{1}-V_{2}$ has been used in deriving Eq. (6). Note, that the mirrors are initially separated at a distance $L_{0}$. The solution of Eq. (6) is found to be

$V_{\mathrm{f}}=V_{0} \cdot v$

with $v=\left(L_{0} / L_{\mathrm{f}}\right)^{\left(\Delta V_{\mathrm{G}} / 2 \Delta V_{\mathrm{S}}\right)}($ Chen 1984; Mann \& Classen 1995). In order to derive a differential equation for the evolution of the pitch angle Eqs. (1)-(4) will be employed taking into account the relationship (5). Then, $\Delta \alpha_{n}=\alpha_{n+1}-\alpha_{n}$ can be calculated to be

$\Delta \alpha_{n}=\arctan \left[(-1) \cdot \frac{\Delta V_{\mathrm{G}}}{V_{n}} \cdot \sin \alpha_{n} \cdot \cos \alpha_{n}\right]$

leading to a differential equation according to $\Delta \alpha_{n} / \Delta t_{n} \Rightarrow$ $\mathrm{d} \alpha / \mathrm{d} t$, i.e.,

$\frac{\mathrm{d} \alpha}{\mathrm{d} t}=-\frac{\Delta V_{\mathrm{G}}}{2\left(L_{0}-\Delta V_{\mathrm{S}} t\right)} \cdot \sin \alpha \cdot \cos \alpha$ 




Fig. 6. Scheme of the area integration of the integral (12) in the $V_{\|}-V_{\perp}$ space.

with the initial condition $\alpha(t=0)=\alpha_{0}$. The solution is given by

$$
\frac{\tan \alpha_{\mathrm{f}}}{\tan \alpha_{0}}=\frac{1}{v}
$$

relating the initial pitch angle $\alpha_{0}$ and final one $\alpha_{\mathrm{f}}$.

Thus, the solutions of Eqs. (6) and (9) describe the evolution of the particle in the velocity space $\left\{V_{\|}(t) ; V_{\perp}(t)=\right.$ $\left.V_{\|} \tan \alpha(t)\right\}$ during the acceleration between $t_{0}=0$ and $t_{\mathrm{f}}=$ $\left(L_{0}-L_{\mathrm{f}}\right) / \Delta V_{\mathrm{S}}$ (see Fig. 6). Here, $V_{\|}$and $V_{\perp}$ denote the particle velocity parallel and perpendicular to the ambient magnetic field, respectively. Now, an ensemble of particles is regarded in the velocity space. All particles initially located on the path determined by $V_{\|}(t) ; V_{\perp}(t)$ in the velocity space are accelerated and receive the final velocity $V_{\mathrm{f}} / \cos \alpha_{\mathrm{f}}$ and final pitch angle $\alpha_{\mathrm{f}}$, i.e., all particles with pitch angles $\alpha_{0} \geq \alpha \geq \alpha_{\mathrm{f}}$ receive an acceleration. Then, the total number of accelerated particles can be calculated by

$\frac{N_{\mathrm{acc}}}{N_{0}}=4 \pi \int_{0}^{\infty} \mathrm{d} V V^{2} f_{\mathrm{v}}(V) \int_{\alpha_{0}}^{\alpha_{\mathrm{f}}} \mathrm{d} \alpha \sin \alpha=\mu_{0}-\mu_{\mathrm{f}}$

with $V=V_{\|} / \cos \alpha, \mu_{0}=\cos \alpha_{0}$, and $\mu_{\mathrm{f}}=\cos \alpha_{\mathrm{f}}$. Here, $f_{\mathrm{v}}$ denotes the distribution function in the velocity space. It is normalized to unity. Now, it is intended to calculate the total number of particles $N_{\text {acc }}\left(\epsilon_{\mathrm{f}}\right)$ accelerated up to a final (maximum) energy $\epsilon_{\mathrm{f}} . \epsilon=E / m_{\mathrm{e}} c^{2}=V^{2} / 2 c^{2}$ ( $m_{\mathrm{e}}$, electron mass; $c$, velocity of light) denotes the kinetic energy $E$ normalized to the rest energy $m_{\mathrm{e}} c^{2}$. Consequently, the final energy $\epsilon_{\mathrm{f}}$ is given by $\epsilon_{\mathrm{f}}=V_{\mathrm{f}}^{2} / 2 c^{2} \cdot \cos ^{2} \alpha_{\mathrm{f}}$. In order to obtain $N_{\mathrm{acc}}\left(\epsilon_{\mathrm{f}}\right)$ the integral (11) must be carried out as an area integral over the dashed region depicted in Fig. 6. This is conveniently done not in the $V_{\|}-V_{\perp}$ space but in the $\epsilon-\mu$ space with $\mu=\cos \alpha$. In carrying out this area integration

$$
\begin{aligned}
\frac{N_{\mathrm{acc}}\left(\epsilon_{\mathrm{f}}\right)}{N_{0}}= & 4 \pi c^{3} \int_{0}^{\epsilon_{0}\left(\epsilon_{\mathrm{f}}\right)} \mathrm{d} \epsilon \sqrt{2 \epsilon} f_{\mathrm{v}}(\epsilon)\left(\mu_{\mathrm{f}}-\mu_{0}\right) \\
& +4 \pi c^{3} \int_{\epsilon_{0}\left(\epsilon_{\mathrm{f}}\right)}^{\epsilon_{\mathrm{f}}} \mathrm{d} \epsilon \sqrt{2 \epsilon} f_{\mathrm{v}}(\epsilon)\left(\mu_{\mathrm{f}}-\mu(\epsilon)\right)
\end{aligned}
$$

results for the total number $N_{\text {acc }}\left(\epsilon_{\mathrm{f}}\right)$ of particles accelerated up to a final energy $\epsilon_{\mathrm{f}}$. In the $\epsilon-\mu$ space the function $\mu(\epsilon)$ defines the path of an electron, which is accelerated from the initial energy $\epsilon_{0}\left(\epsilon_{\mathrm{f}}\right)$ with an initial pitch angle $\alpha_{0}$ (or $\left.\mu_{0}\right)$ up to the final one $\epsilon_{\mathrm{f}}$ with the final pitch angle $\alpha_{\mathrm{f}}$ (or $\mu_{\mathrm{f}}$ ) by the considered mechanism. By means of Eqs. (7) and (10) the initial energy $\epsilon_{0}$ is related to the final one $\epsilon_{\mathrm{f}}$ by

$\epsilon_{0}\left(\epsilon_{\mathrm{f}}\right)=\epsilon_{\mathrm{f}} \cdot \frac{1}{v^{2}} \cdot\left(\frac{1+v^{2} \tan ^{2} \alpha_{\mathrm{f}}}{1+\tan ^{2} \alpha_{\mathrm{f}}}\right)$

resulting to $\epsilon_{0} / \epsilon_{\mathrm{f}}=\sin ^{2} \alpha_{\mathrm{f}}$ in the case $v \gg 1$ (Chen 1984). Furthermore, $\mu(\epsilon)$ can be deduced to be

$\mu(\epsilon)=\sqrt{\frac{\epsilon-\epsilon_{0} \sin ^{2} \alpha_{0}}{\epsilon}}$

from Eqs. (7) and (10). Finally, the particle distribution function $F_{\epsilon}$ in the energy space can be found by

$F_{\epsilon}(\epsilon)=\frac{\mathrm{d}}{\mathrm{d} \epsilon}\left[\frac{N_{\mathrm{acc}}(\epsilon)}{N_{0}}\right]$

for the accelerated particles. This expression will be employed to compute the differential fluxes of energetic electrons at CIR related shocks as discussed in the next section. This approach has recently been generalized for relativistic electrons by Mann et al. (2001).

\section{Discussion}

Now, the mechanism of electron acceleration as introduced in the previous Section is used to explain the correlation between the ratios $\lg \left(j_{\text {shock }} / j_{0}\right)$ and the jump of the magnetic field $B_{2} / B_{1}$ as deduced for CIR related shocks from the HISCALE data (see Fig. 2).

The differential flux $j(E)$ is related to the distribution function $f_{\mathrm{p}}(p)$ in the momentum space by $j(E)=N_{0} \cdot f_{\mathrm{p}}(p) \cdot p^{2}$ (Landau \& Lifschitz 1975). Here, the distribution function $f_{\mathrm{p}}(p)$ is normalized to unity. If $f_{\mathrm{E}}(E)$ denotes the distribution function in the energy space, the conservation of the particle number density in the phase space, i.e., $N_{0} f_{\mathrm{p}}(p) d^{3} p=$ $4 \pi N_{0} p^{2} f_{\mathrm{p}}(p) \mathrm{d} p=4 \pi j(E) \mathrm{d} p=N_{0} f_{\mathrm{E}}(E) \mathrm{d} E$, leads to

$j(E)=\frac{N_{0}}{4 \pi} \cdot \sqrt{\frac{2 E}{m}} \cdot f_{\mathrm{E}}(E)$.

Here $N_{0}$ denotes the undisturbed particle number density. Then, the distribution function $f_{\mathrm{E}}$ in the energy space is related to that $f_{\mathrm{v}}$ in the velocity space by $f_{\mathrm{E}}(\epsilon)=\left(4 \pi c^{3} / m_{\mathrm{e}} c^{2}\right) \cdot(2 \epsilon)^{1 / 2} \cdot f_{\mathrm{v}}(\epsilon)$.

Now the flux $j_{\text {shock }}$ of accelerated electrons at the shock crossing is compared with that $j_{0}$ in the undisturbed upstream region. In order to do this a so-called kappa distribution is assumed to exist for velocity distribution function $f_{\mathrm{v}}(\epsilon)$ in the undisturbed solar wind, i.e. upstream of CIR related shock waves. A kappa distribution is defined by

$f_{\mathrm{v}}(E)=C_{\kappa}\left[1+\frac{E}{\kappa E_{\kappa}}\right]^{-\kappa-1}$

with the normalization constant

$C_{\kappa}=\frac{1}{2 \pi} \cdot\left(\frac{m}{2 \kappa E_{\kappa}}\right)^{3 / 2} \cdot \frac{\Gamma(\kappa+1)}{\Gamma(3 / 2) \cdot \Gamma(\kappa-1 / 2)}$ 
(Krauss-Varban \& Burgess 1991). It behaves like a Maxwellian distribution for $E \leq E_{K}$ and a power law one for $E \gg E_{\kappa}$. The temperature $T$ can be defined in a kinetic manner, i.e. as the mean energy $\bar{E}$ of an ensemble average with respect to the kappa distribution, resulting in

$\bar{E}=\frac{3}{2} \cdot E_{\kappa} \cdot \frac{\kappa}{(\kappa-3 / 2)}=\frac{3}{2} \cdot k_{\mathrm{B}} T$

( $k_{\mathrm{B}}$, Boltzmann's constant). Thus, the kappa distribution (see Eq. (17)) is unambiguously determined by fixing the temperature $T$ and the parameter $\kappa$. Inserting the distribution function (Eq. (17) into Eq. (16))

$j_{0}(E)=\frac{N_{1} c^{4}}{m_{\mathrm{e}} c^{2}} \cdot C_{\kappa, 1} \cdot 2 \epsilon \cdot\left[1+\frac{\epsilon}{\kappa \epsilon_{\kappa, 1}}\right]^{-\kappa-1}$

results for the undisturbed flux with $\epsilon_{\kappa}=E_{\kappa} / m_{\mathrm{e}} c^{2}$. Consequently, $N_{1}$ denotes again the upstream particle number density. Here, the quantities $C_{\kappa, 1}$ and $\epsilon_{\kappa, 1}$ are determined by the temperature in the upstream region. In particular, the $3 \mathrm{D}$ plasma instrument aboard the Wind spacecraft is able to measure the electron distribution function under quiet solar wind conditions in the heliosphere at $1 \mathrm{AU}$. These measurements can be fitted by kappa distributions with $\kappa=2.5-3.5$ (Lin et al. 1996). Thus, a kappa distribution with $T=1 \times 10^{5} \mathrm{~K}$ (see Sect. 2) and $\kappa=2.5$ is assumed to be an appropriate electron distribution in the upstream region of CIR related shock waves, although the CIRs occur mainly at heliospheric distances of 4-5 AU.

The flux of the accelerated electrons at the shock crossing is determined by

$j_{\text {shock }}=\frac{N_{2} c}{4 \pi m_{\mathrm{e}} c^{2}} \cdot \sqrt{2 \epsilon} \cdot F_{\epsilon}(\epsilon)$

taking into account $f_{\mathrm{E}}(\epsilon)=\left(N_{2} / N_{\text {acc }}\right) \cdot\left(F_{\epsilon}(\epsilon) / m_{\mathrm{e}} c^{2}\right) . F_{\epsilon}(\epsilon)$ is numerically calculated by means of Eqs. (12)-(15). Then, the kappa distribution of the downstream region must be inserted into Eq. (12), i.e. $\kappa \epsilon_{\kappa}=(\kappa-3 / 2) \cdot k_{\mathrm{B}} T_{2} / m_{\mathrm{e}} c^{2}$ is to be inserted.

Now the results theoretically obtained from the presented electron acceleration mechanism are compared with the observations summarized in Fig. 2. In order to do this the plasma parameters usually found upstream of CIR related shocks (see Sect. 2) will be employed. The jumps of the particle number density $N_{2} / N_{1}$, of the magnetic field $B_{2} / B_{1}$, and of the temperature $T_{2} / T_{1}$ are related to the Alfven-Mach number $M_{\mathrm{A}}$ by the well-known Rankine-Hugoniot relations (Kennel et al. 1985) as depicted in Fig. 7. Here, a plasma beta of 0.8 and an angle $\theta_{B, n}=54^{\circ}$ (see Sect. 2) have been adopted. Using Eqs. (20) and (21) the ratio $j_{\text {shock }} / j_{0}$ can be expressed by

$\frac{j_{\text {shock }}}{j_{0}}=\frac{1}{4 \pi c^{3}} \cdot \frac{N_{2}}{N_{1}} \cdot \frac{F_{\epsilon}(\epsilon)}{\sqrt{2 \epsilon} \cdot C_{\kappa, 1}} \cdot\left[1+\frac{\epsilon}{\kappa \epsilon_{\kappa, 1}}\right]^{\kappa+1}$.

Firstly, it is seen that the jump of the density $N_{2} / N_{1}$ and the temperature $T_{2} / T_{1}$ appear in the second factor of the ratio $j_{\text {shock }} / j_{0}$ and in the ratio of the normalization constants $C_{\kappa, 2} / C_{\kappa, 1}$, respectively. Thus, a variation of these shock parameters influences immediately the ratio $j_{\text {shock }} / j_{0}$. Furthermore,

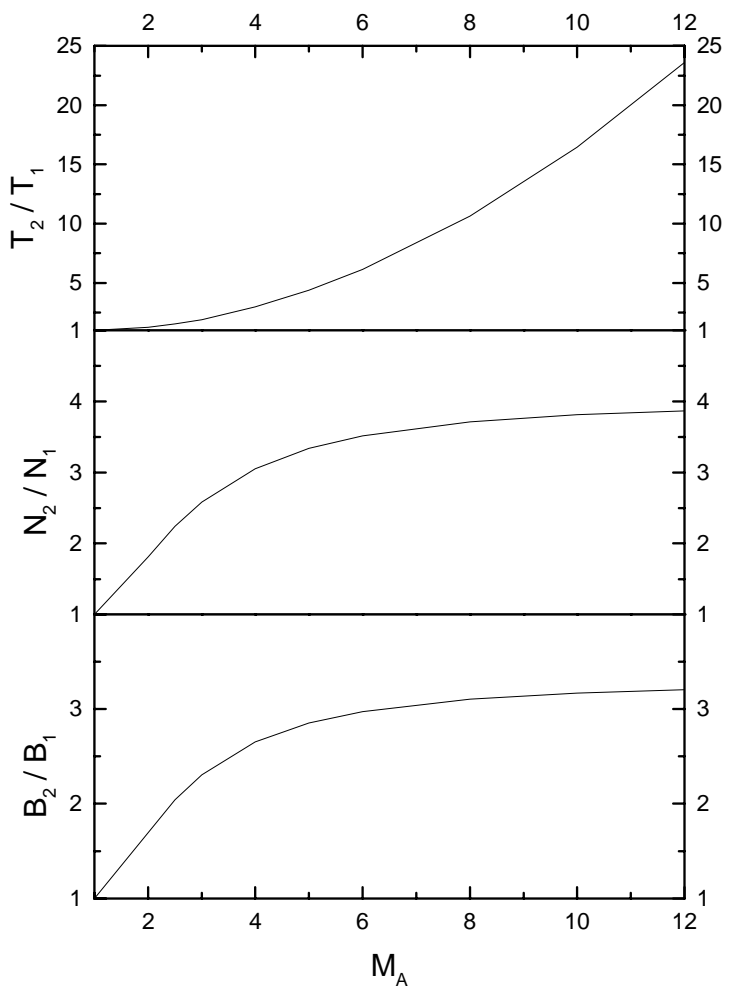

Fig. 7. Dependence of the jump of the temperature $T_{2} / T_{1}$, the particle number density $N_{2} / N_{1}$, and the magnetic field $B_{2} / B_{1}$ across the shock on the Alfven-Mach number $M_{\mathrm{A}}$ according to the Rankine-Hugoniot relationships (Kennel et al. 1985). An angle $\theta_{B, n}=54^{\circ}$ between the upstream magnetic field and the shock normal, a plasma beta $\beta=0.8$ and an adiabatic index of $5 / 3$ has been used.

the variation of the jump of the magnetic field $B_{2} / B_{1}$ influences this ratio in another way. As already mentioned large amplitude magnetic field fluctuations appear in the vicinity of the shock transition. Here, the maximum of the magnetic field compression is assumed to be $B_{\max } / B_{1}=1.4 B_{2} / B_{1}$, for example (Kennel et al. 1985). Then, the final pitch angle is given by $\alpha_{\mathrm{f}}=\arcsin \left[\left(1.4 B_{2} / B_{1}\right)^{-1 / 2}\right]$. Furthermore, the distance between the two mirrors is assumed to be diminished from $L_{0}=50 d_{\mathrm{i}}$ up to $L_{\mathrm{f}}=10 d_{\mathrm{i}}$. The velocities $V_{1}$ and $V_{2}$ of the mirrors $M 1$ and $M 2$ are regarded to be $V_{1}=M_{\mathrm{A}} \cdot v_{\mathrm{A}}$ and $V_{2}=\left[1+\left(M_{\mathrm{A}}-1\right) / 2\right] \cdot v_{\mathrm{A}}$, i.e. $\Delta V_{\mathrm{S}}=V_{1}-V_{2}=\left(M_{\mathrm{A}}-1\right) v_{\mathrm{A}} / 2$, leading to $\Delta V_{\mathrm{G}}=2 \cdot \Delta V_{\mathrm{S}} \cdot \sec \left(54^{\circ}\right)\left(\theta_{1} \approx \theta_{2}=54^{\circ}\right)$ and $v=\left(L_{0} / L_{\mathrm{f}}\right)^{\left(\Delta V_{\mathrm{G}} / 2 \Delta V_{\mathrm{S}}\right)}=15.46$ as well as to the initial pitch angle $\alpha_{0}=\arctan \left[v \cdot \tan \left(\alpha_{\mathrm{f}}\right)\right]$ (see Eq. (10)). Because of $v=15.46 \gg 1$ Eq. (13) reduces to $\epsilon_{\mathrm{f}} / \epsilon_{0}=1.4 \cdot B_{2} / B_{1}$. All quantities just described are directly or indirectly influenced by the variation of the jump $B_{2} / B_{1}$ of the magnetic field. In Fig. 2 the full line represents the dependence of the ratio $j_{\text {shock }} / j_{0}$ numerically calculated by Eq. (22) on the jump of the magnetic field $B_{2} / B_{1}$ taking into account the results of the RankineHugoniot relations (see Fig. 7). As evidently seen in Fig. 2 the theoretically obtained results can explain the correlation deduced from the data provided by the HISCALE instruments aboard the ULYSSES spacecraft. 


\section{Summary}

The study of the enhancements $j_{\text {shock }} / j_{0}$ of energetic electron fluxes in the range $30-40 \mathrm{keV}$ during the crossing of CIR related shocks reveals a relationship between the ratio $j_{\text {shock }} / j_{0}$ and the jump $B_{2} / B_{1}$ of the magnetic field of the associated shock as shown in Fig. 2. This relationship has been explained by an electron acceleration mechanism, which acts as an interaction of electrons with the large amplitude magnetic field fluctuations in the vicinity of the shock transition. The enhancements of the energetic electron fluxes are caused by the heating of the electrons as a result of the shock crossing and the subsequent acceleration due to their interaction with the magnetic field fluctuations in the downstream region. Since the large amplitude magnetic field fluctuations, which are necessary for the electron acceleration, appear mainly in the vicinity of the shock transition for quasi-perpendicular shocks, the proposed acceleration mechanism acts very locally and fast for electrons at spatial and temporal scales of few ion inertial lengths and inverse proton cyclotron frequencies, respectively. In contrast to the shock drift acceleration (Holman \& Pesses 1983; Krauss-Varban \& Wu 1989; Kraus-Varban et al. 1989), where the energy gain is limited because of a single shock encounter and only efficient at nearly perpendicular shocks, the mechanism presented is much more efficient since the electrons accumulate energy due to the multiple reflections at the large amplitude magnetic field fluctuations. Furthermore, this mechanism is a deterministic one, unlike diffuse shock acceleration (Axford et al. 1977), which is a stochastical process acting in the whole up- and downstream region of the associated shock. Recently, Classen et al. (1999) reported on a high correlation between the fluxes of $1 \mathrm{MeV}$ protons and the low frequency magnetic field turbulence in the downstream region of CIR related shocks, especially in the vicinity immediately after the shock transition. This result implies that wave-particle interactions in the downstream region even after the shock transition play an important role for acceleration of particles at CIR-related shock waves. This observational result confirms additionally the acceleration mechanism proposed in this paper.

\section{References}

Anderson, K. A. 1969, J. Geophys. Res., 73, 2387

Axford, W. I., Leer, E., \& Skadron, G. 1977, Proc. Int. Conf. Cosmic Rays 15 th, 11, 132

Balogh, A., Beek, T. J., Forsyth, J. R., et al. 1992, A\&AS, 92, 271 T

Balogh, A., Gonzalez, J. A., Forsyth, R. J., et al. 1995, Space Sci. Rev., 72,171

Bame, S. J., McComas, D. J., Barraclough, B. I., et al. 1992, A\&AS, 92, 237

Bame, S. J., Goldstein, B. E., Gosling, J. T., et al. 1993, Geophys. Res. Lett., 20, 2323

Barnes, C. W., \& Simpson, J. A. 1976, ApJ, 210, L91

Cairns, I. H., \& Robinson, R. D. 1987, Solar Phys., 111, 356

Cane, H. V., Stone, R. G., Fainberg, J. L., Stewart, R. T., \& Steinberg, J. L. 1981, Geophys. Res. Lett., 8, 1285
Chen, F. F. 1984, Introduction to Plasma Physics and Controlled Fusion: Plasma Physics vol. 1 (Kluwer, Dordrecht), 30

Classen, H.-T., Mann, G., \& Keppler, E. 1998, A\&A, 335, 1001

Classen, H.-T., Mann, G., Forsyth, R. J., \& Keppler, E. 1999, A\&A, 347,313

Decker, R. B. 1988, Space Sci. Rev., 48, 195

Desai, M. I., Marsden, R. G., Sanderson, T. R., et al. 1998, J. Geophys. Res., 103, 2, 003

Fermi, E. 1949, Phys. Rev., 75, 1149

Gosling, J. T., \& Pizzo, V. J. 1999, in Corotating Interaction Regions, ed. A. Balogh, J. T. Gosling, J. R. Jokipii, R. Kallenbach, \& H. Kunow (Kluwer Academic Publishers, Dordrecht), 21

Gosling, J. T., Bame, S., Feldman, W. C., et al. 1993, Geophys. Res. Lett., 21, 2335

Holman, G. D., \& Pesses, M. E. 1983, ApJ, 267, 837

Kennel, C. F., Edmiston, J. P., \& Hada, T. 1985, in Collisionless Shocks in the Heliosphere: A Tutorial Review, ed. B. T. Stone, \& R. G. Stone, Geophysical Monographs GN-34 (AGU, Washington DC), 1

Keppler, E., Drolias, B., Fraenz, M., et al. 1996, A\&A, 316, 464

Krauss-Varban, D., \& Wu, C. S. 1989, J. Geophys. Res., 94, 15, 367

Krauss-Varban, D., Burgess, D., \& Wu, C. S. 1989, J. Geophys. Res., 94, 15, 089

Krauss-Varban, D., \& Burgess, D. 1991, J. Geophys. Res., 96, 143

Landau, L. D., \& Lifshitz, E. M. 1975, The classical field theory of fields (Pergamon Press, Oxford), 264

Lanzerotti, L. J., Gold, R. E., Anderson, K. A., et al. 1992, A\&AS, 92, 349

Lin, R. P., Larson, D., McFadden, J., et al. 1996, Geophys. Res. Lett., 23, 1211

Lopate, N. 1989, J. Geophys. Res., 94, 9, 995

Mann, G. 1995, J. Plasma Phys., 53, 109

Mann, G. 1999, in Corotating Interaction Regions, ed. A. Balogh, J. T. Gosling, J. R. Jokipii, R. Kallenbach, \& H. Kunow (Kluwer Academic Publishers, Dordrecht), 389

Mann, G., \& Classen, H. T. 1995, A\&A, 304, 576

Mann, G., Lühr, H., \& Baumjohann, W. 1994, J. Geophys. Res., 99, 13,315

Mann, G., Classen, H.-T., \& Motschmann, U. 2001, J. Geophys. Res., $106,25,323$

Marsden, R. G., Smith, E. J., Cooper, J. F., \& Tranquille, C. 1996, A\&A, 316, 279

Mason, G. M., \& Sanderson, T. R. 1999, in Corotating Interaction Regions, ed. A. Balogh, J. T. Gosling, J. R. Jokipii, R. Kallenbach, \& H. Kunow (Kluwer Academic Publishers, Dordrecht), 77

McDonald, F. B., Teegarden, B. J., Trainor, J. H., von Rosenvinge, T. T., \& Weber, W. R. 1976, ApJ, 203, L149

Neugebauer, M. C., \& Snyder, C. W. 1966, J. Geophys. Res., 71, 4469 Parker, E. N. 1958, ApJ, 128, 664

Pizzo, V. J. 1978, J. Geophys. Res., 83, 5, 563

Roelof, E. C., Simnett, G. M., \& Tappin, S. J. 1966, A\&A, 316, 481

Scarf, F. L., Fredricks, R. W., Frank, L. A., \& Neugebauer, M. 1971, J. Geophys. Res., 76, 5162

Schwenn, R. 1990, in Physics of the Inner Heliosphere, ed. R. Schwenn, \& E. Marsch (Springer Verlag, Berlin), 99

Simpson, J. A., et al. 1992, A\&AS, 92, 365

Simnett, G. M., \& Roelof, E. C. 1995, Space Sci. Rev., 72, 302

Toptygin, I. N. 1980, Space Sci. Rev., 26, 157

Tsurutani, B. T., \& Lin, R. P. 1985, J. Geophys. Res., 90, 1

Wild, J. P., \& McCready, L. L. 1950, Austr. J. Sci. Res., Ser. A, 3, 387 